\title{
Optical properties of Photonic Crystal Fibres with the strain
}

\author{
J.M. Lázaro, C. Galindez, A. Quintela, A. Cobo and J.M. López-Higuera \\ Photonic Engineering Group-University of Cantabria, \\ Avda. Los Castros s/n - c.p. 39005 Santander, Spain
}

\begin{abstract}
The effective index, fundamental mode width, numerical aperture are some important parameters of a fibre. The behaviour of these parameters for a Modified Total Internal Reflection (MTIR) Photonic Crystal Fibre (PCF) is studied when strain is applied. We chose a range of normalized frequency $(\Lambda / \lambda)$ and a range of $d / \Lambda$ and we analyzed the behaviour for different values of strain. The sensibilities of the studied parameter are obtained in the chosen range. Finally, the region of maximum influence of the strain is observed.
\end{abstract}

Keywords: Fibre characterization, Photonic Crystal Fibre, effective index, fundamental mode width, numerical aperture, half-divergence angle, sensitivity to strain.

\section{INTRODUCTION}

Photonic Crystal Fibres (PCF) are a new type of fibres with special properties [1-5]. In the last year, the investigation in this new type of fibres is increasing. These PCFs are fibres of pure silica formed by a periodic holes structure in the transverse section along the length of the fibre. The periodicity is broken for the absence of one hole or a hole with bigger diameter in the centre of the transverse section, enabling the propagation of the light along the PCF length.

Two different propagation mechanisms, depending on the design of the periodic structure, are already demonstrated [6]. Like in the telecommunication standard fibres, in a type of these PCFs the effective cladding index is lower than a solid core due to the air holes in the cladding, producing the guide of the light in the core. This type of guide is called modified total internal reflection (MTIR) and the periodicity is not necessary [7]. On the other hand, in photonic bandgap PCFs, light can be confined inside a low-index region - even a large air hole - by reflection from the periodic transversal structure of the cladding [8]. In this case, modes of propagations do not exist for certain wavelengths along the transverse section of the PCFs, being the light confined in their interior.

Due to their flexibility or behaviour design capacity, PCFs have been intensively explored for a wide range of applications. These include optical fibre communications [1-5], trap or guide of particles [9] and, of course, in fibre sensors [10]. In some kind of the previous, the PCF is used as transducer and, in these cases, the higher as possible sensitivity to the external desirable parameters (pressure, temperature, strain, etc) is highly desirable.

In this paper, we study the MTIR-PCF and the sensitivity of several optical parameters to strain is analyzed. The range of normalized frequency of maximum sensitivity and the optimum structural fibre parameter are easily identified. These results are very useful for optimum design of the MTIR-PCF based fibre transducer element.

\section{THEORETICAL PRINCIPLES}

In figure 1 it is shown the structure of the analyzed PCF. It is composed for two main parameters: the period or holes distance $(\Lambda)$ and the holes diameter (d). In this fibre there is a parameter that relates these other parameters, this is the air fraction $\left(\mathrm{f}=\pi /(2 \cdot \sqrt{3}) \cdot(\mathrm{d} / \Lambda)^{2}\right)$ that is defined as the area of the air holes relative to the total hexagonal structured area.

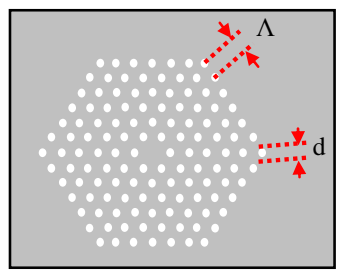

Fig. 1. Transversal section illustration of the MTIR-PCF.

Third European Workshop on Optical Fibre Sensors, Antonello Cutolo, Brian Culshaw, José Miguel López-Higuera, Eds., Proceedings of SPIE Vol. 6619, 661935, (2007) · 0277-786X/07/\$18 - doi: 10.1117/12.738621 
In this paper, we analyze the behavior of the effective index ( $\mathrm{n}_{\mathrm{eff}}$ ), fundamental mode width (w), numerical aperture (NA) and half-divergence angle $(\theta)$ in the MTIR-PCF when strain is applied. For it, we have to consider two variations in the PCF: structural variation and silica index variation. In the case of the structure variation we have a decrease of the period and the holes diameter with the increase of strain. As the volume remains constant and considering a perfect cylindrical form of the holes, we can calculate the new holes radius in the strain region applying the following expression:

$$
\pi \cdot r_{1}^{2} \cdot h_{1}=\pi \cdot r_{2}^{2} \cdot h_{2} \Rightarrow r_{2}=r_{1} \cdot \sqrt{\frac{h_{1}}{h_{2}}}
$$

where $r_{1}$ and $h_{1}$ are the radius and the length without strain and $r_{2}$ and $h_{2}$ are the radius and the length with strain. Beside, as the ratio $d / \Lambda$ can be considered constant when strain is applied we can calculate the new period by means of the expression:

$$
\Lambda_{2}=\frac{1}{d / \Lambda} \cdot d_{2} \quad \text { con } \quad d_{2}=2 \cdot r_{2}
$$

On the other hand, there is a change in the refractive index of the silica $\left(\Delta \mathrm{n}_{\mathrm{SI}}\right)$ if a section of the fibre with length $\mathrm{L}$ is elongated to $\mathrm{L}+\Delta \mathrm{L}$ due to the strain. This change can be calculated with the expression:

$$
\Delta n_{S i}=-0.5 \cdot n_{S i}^{3} \cdot\left(\rho_{12}-v \cdot\left(\rho_{11}+\rho_{12}\right)\right) \frac{\Delta L}{L}
$$

where $\rho_{12}=0.252, \rho_{11}=0.113$ are the strain-optic tensor coefficients, $v=0.17$ (@25 ${ }^{\circ} \mathrm{C}$ ) is the Poisson's ratio and $\Delta \mathrm{L}$ is the elongation.

Using the previous results, the desired parameters with and without strain and found the difference can be calculated. The effective index is just the ratio of the modulus of the wavevector $\mathrm{k}$ and the mode frequency. For the calculation of the fundamental mode width the second moment definition has been used:

$$
w=\sqrt{f_{j} \cdot A_{j}}
$$

where $f_{\mathrm{j}}$ is the fraction of the field energy in component $\mathrm{j}$ and $A_{\mathrm{j}}$ is the mode area for each component defined as the field-average of the position vector:

$$
A_{j}=\langle\vec{x} \cdot \vec{x}\rangle_{j}-\langle\vec{x}\rangle_{j}^{2}
$$

In this paper the simulations of the effective index and the fundamental mode width have been realized with the Bandsolve packet from Rsoft. The numerical aperture have been calculated with the results of the mode width by mean of the expression:

$$
N A=\sin (\theta) \approx\left(1+\left(\frac{\pi \cdot w}{\lambda}\right)^{2}\right)^{-\frac{1}{2}}
$$

where $\theta$ is the divergence angle.

\section{RESULTS AND ANALYSIS}

We have studied the variation of the parameters previously indicated, due to the strain, for different values of the normalized frequency $(\Lambda / \lambda)$ in function of $d / \Lambda$. It gives as a range of values of $\Lambda / \lambda$ and $d / \Lambda$ that is more sensitive. This analysis has been realized for several values of strain $(\varepsilon)$. Y. Zhu [11] have published that a case of this type of fibre tolerates a maximum strain of $2.75 \% €$, for this reason we analyzed a range between 0.5 to 2.5 with step 0.5 . In figure 2 we show an example of the maximum difference of the values of effective index with strain minus the values without strain in function of the applied strain for different values of $d / \Lambda$. We can observe a linear behaviour with the strain with different slope depending of $\mathrm{d} / \Lambda$ (in this case the effective index difference is negative due to the effective index decrease with the strain). 


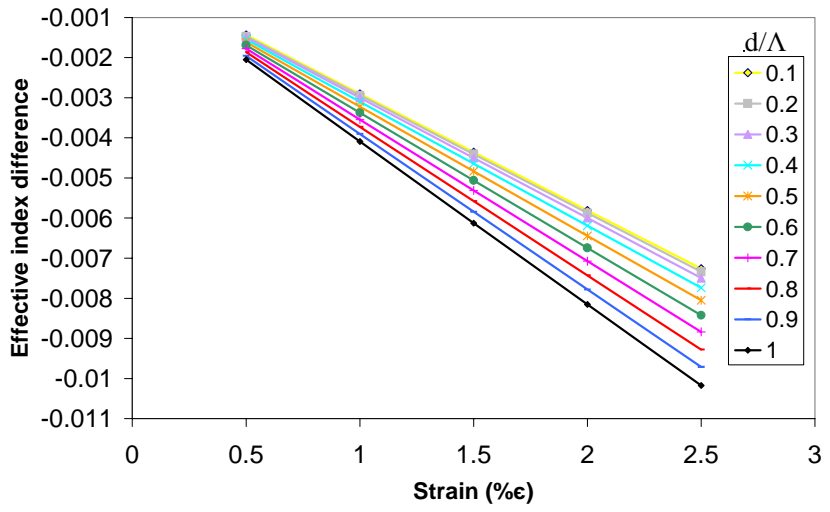

Fig. 2. Effective index difference in function of the applied strain for different values of $d / \Lambda$.

Next the obtained results for the analyzed parameters are shown. We have obtained the behaviour of the analyzed parameters without strain and the sensitivity, in both cases, in function of the normalized frequency for different values of $d / \Lambda$. The sensitivity is defined as the variation of each parameter divided by the applied strain in percentage. Figures 3, 4 and 5 show the results for the effective index, fundamental mode width and numerical aperture, respectively.

It can be seen that different values of the normalized frequency and $d / \Lambda$ give different values of the analyzed optical parameter and sensitivity. In this way, depending on the fibre structural parameters ( $\Lambda$ and $d)$ and the wavelength we will obtain a different value of effective index, fundamental mode width and numerical aperture. When strain is applied, these values will change according to a sensitivity gives also by the chosen $\Lambda, d$ and $\lambda$.
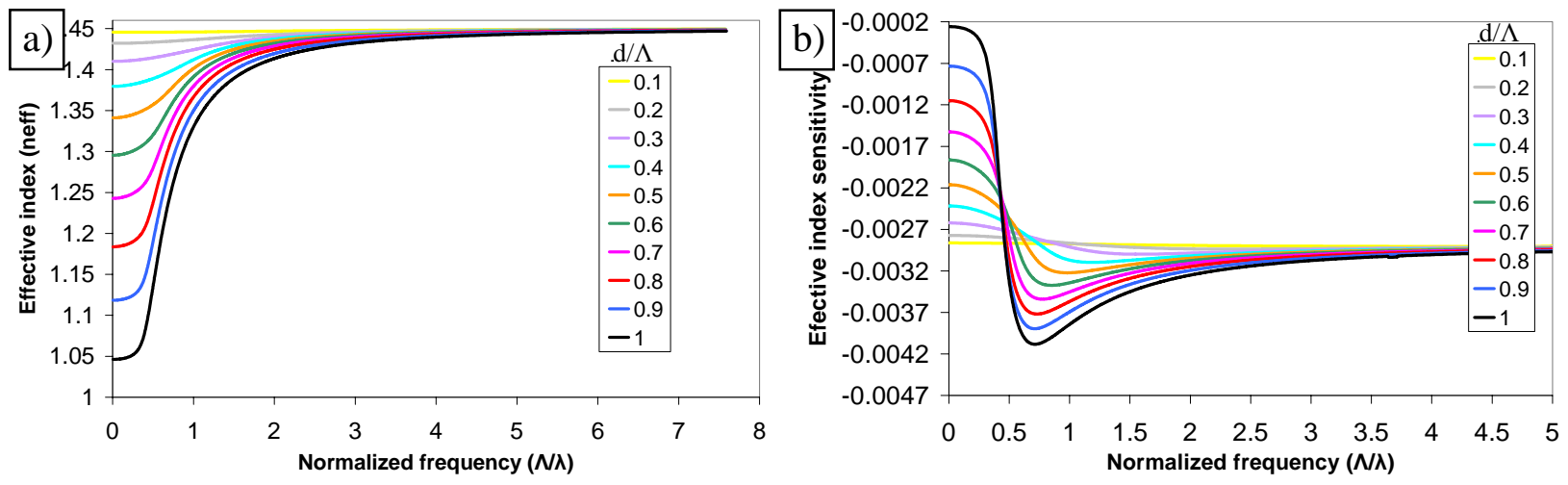

Fig. 3. Effective index behaviour for different : a) without strain, b) sensitivity.
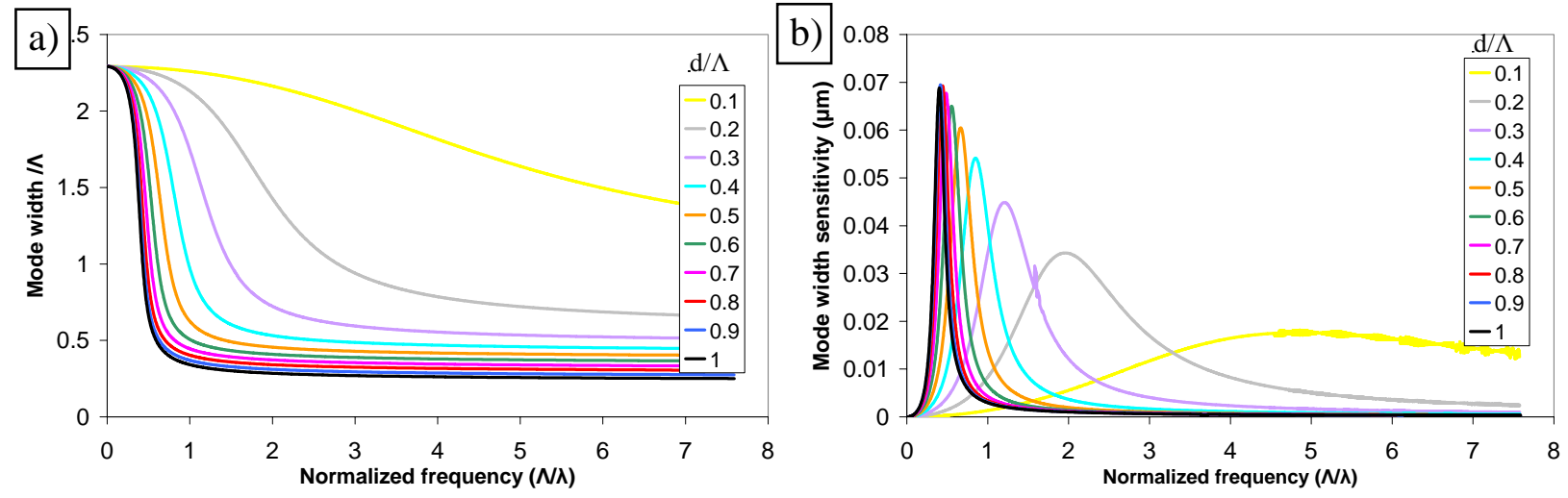

Fig. 4. Fundamental mode width behaviour for different $d / \Lambda$ : a) without strain, b) sensitivity. 

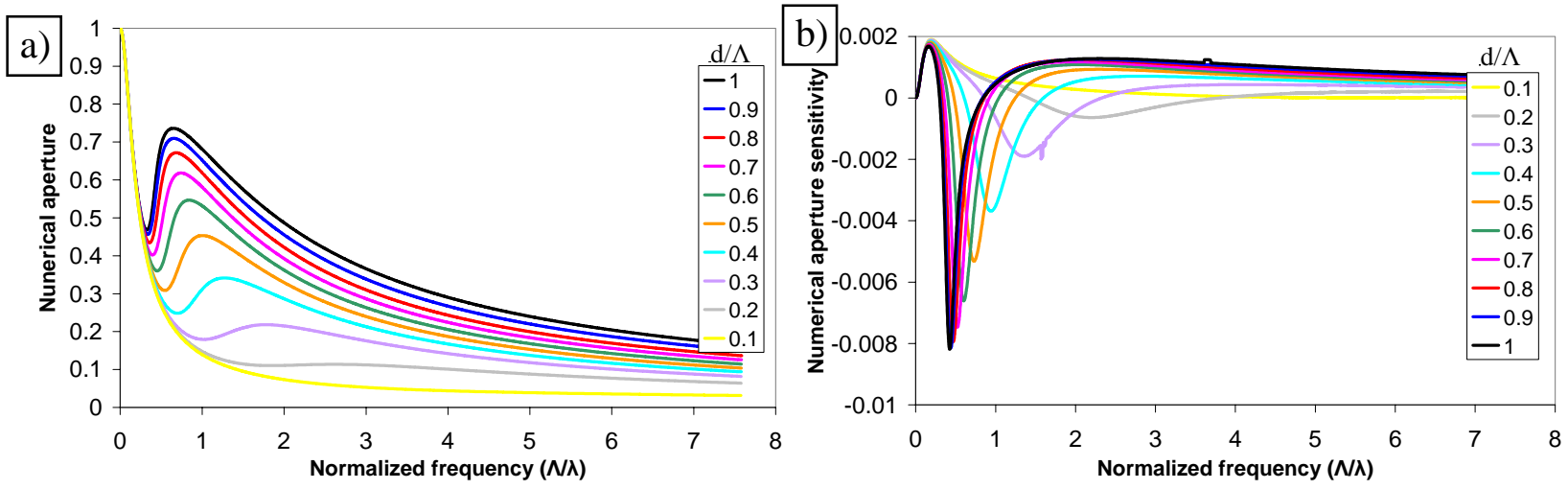

Fig. 5. Numerical aperture behaviour for different $d / \Lambda$ : a) without strain, b) sensitivity.

Although the sensitivity curves form are different, it can be observed that in all the cases the sensitivity has different values for small normalized frequency and tend to a constant value when the normalized frequency grows. In this way, in all the cases the maximum sensitivity values are obtained for $\Lambda / \lambda$ small (considering the maximum sensitivity as the maximum value in module because the sign only indicate if the parameter increase or decrease when strain is applied). Also we have obtained that with bigger values of $\mathrm{d} / \Lambda$ we obtain bigger sensitivity.

\section{CONCLUSIONS}

We have studied the behaviour of the effective index, fundamental mode width, and numerical aperture of a Modified Total Internal Reflection (MTIR) Photonic Crystal Fibre (PCF) when strain is applied. The range of strain studied is between 0.5 to $2.5 \% \varepsilon$. We have observed that the variation of these parameters can be approached by a linear function with the strain. It is also obtained the sensitivity of these parameters for a range of the normalized frequency $(\Lambda / \lambda)$ and $\mathrm{d} / \Lambda$. The values of $\mathrm{d} / \Lambda$ and normalized frequency which produces bigger sensitivity was calculated. These values appear at large $d / \Lambda$ and in the small normalized frequency $(\Lambda / \lambda)$ ranges.

\section{ACKNOWLEDGEMENTS}

This work was supported in part by TEC'2004-05936-C02-02 and TEC’2005-08218-C02-02 projects.

\section{REFERENCES}

1. J.C. Knight, T.A. Birks, P.S.J. Russell, and D.M. Atkin, "All-silica single mode optical fiber with photonic crystal cladding," Opt. Lett. 21, 1547-1549 (1996).

2. T.A. Birks, J.C. Knight, and P.S.J. Russell, "Endlessly single-mode photonic crystal fiber," Opt. Lett. 22, 961-963 (1997).

3. J. Broeng, D. Mogilevstev, S.E. Barkou, and A. Bjarklev, "Photonic crystal fibers: A new class of optical waveguides," Optical Fiber Technology 5, 305-330 (1999).

4. J.C. Knight, T.A. Birks, R.F. Cregan, P.S.J. Russell, and J.P. Sandro, "Photonic crystals as optical fibersphysics and applications," Optical Materials 11, 143-151 (1999).

5. R.S. Windeler, J.L. Wagener, and D.J. DiGiovanni, "Silica-air microstructured fibers: Properties and applications," Optical Fiber Communications conference, San Diego(1999).

6. A. Bjarklev, J. Broeng and A. S. Bjarklev, "Photonic Crystal Fibres”, Kluwer Academic Publishers (2003)

7. T.M. Monro, P.J. Bennett, N.G.R. Broderick and D.J. Richardson, "Holey fibers with random cladding distributions" Opt. Lett. 25, 206-8 (2000).

8. J.C. Knight, J. Broeng, T.A. Birks and P. St. J. Russell, "Photonic band gap guidance in optical fibers" Science 282, 1476-8 (1998).

9. M.J. Renn, R. Pastel, and H.J. Lewandowski, "Laser Guidance and Trapping of Mesoscale Particles in Hollow-Core Optical Fibers”, Physical Review Letters 82 (7), 1574-7 (1999) .

10. J.M. Fini,“Microstructure fibres for optical sensing in gases and liquids”, Meas. Sci. Technol. 15, 1120 (2004).

11. Y. Zhu, P. Shum, H. Bay, M. Yan, X. Yu, J. Hu, J. Hao and C. Lu, "Strain-insensitive and high-temperature longperiod gratings inscribed in photonic crystal fiber”, Optics Letters 30(4), (2005). 